\title{
Therapeutic Anticoagulation in the Presence of a High-Grade Abdominal Solid Organ Injury: A Challenging Case to Manage - Case Report and Literature Review
}

\author{
Yousif Habib Alabboudi ${ }^{a} \quad$ Alaa Haitham Salman $^{b}$ Yousif Hussein El Tayeb ${ }^{a}$ \\ Ali Khammas Yammahi ${ }^{a}$ \\ ${ }^{a}$ General Surgery Department, Rashid Hospital, Dubai Health Authority, Dubai, United Arab Emirates; ${ }^{\mathrm{b}}$ Internship, \\ Rashid Hospital, Dubai Health Authority, Dubai, United Arab Emirates
}

\section{Keywords}

Therapeutic anticoagulation · Trauma · Splenic injury · Prophylactic anticoagulation · High-grade solid organ injury

\begin{abstract}
There are well-established protocols for venous thromboembolism prophylaxis in many patients with a variety of medical and surgical conditions. However, no studies have been published regarding therapeutic anticoagulation in polytrauma patients, who usually present with multiple injuries including vascular injuries and solid organ injuries, which would require therapeutic anticoagulation that increases the risk of bleeding from abdominal solid organ injuries. Here, we present the case of a 45-year-old male, who had a motor vehicle collision and was found to have a grade III splenic injury and had to be started on therapeutic anticoagulation due to popliteal artery injury repair. Our patient was anticoagulated with low-molecular-weight heparin. He had no bleeding complications, and his splenic injury was successfully managed nonoperatively.
\end{abstract}

(C) 2022 The Author(s).

Published by S. Karger AG, Basel

karger@karger.com www.karger.com/dmj

Karger $\stackrel{\text { ' }}{5}$
(C) 2022 The Author(s)

Published by S. Karger AG, Basel

This is an Open Access article licensed under the Creative Commons Attribution-NonCommercial-4.0 International License (CC BY-NC) (http://www.karger.com/Services/OpenAccessLicense), applicable to the online version of the article only. Usage and distribution for commercial purposes requires written permission.

\section{Introduction}

Deep vein thrombosis (DVT) prophylaxis is an established practice in medical and surgical patients, with welldefined and documented protocols published in the world literature over the past years. There are well-established guidelines for DVT prophylaxis for cancer patients [1], as well as for patients undergoing gynecologic [2], orthopedic [3], and bariatric surgeries [4].

Over the past years, new literature was published regarding DVT prophylaxis in the trauma cohort of patients with focus on abdominal solid organ injuries such as the spleen and liver [5, 6]. Lately in 2020, the Western Trauma Association (WTA) published a long-awaited guideline for the prophylaxis of DVT in trauma patients [7].

But up to this date, there are no publications or studies on therapeutic anticoagulation (TAC) in polytrauma patients, keeping in mind that the usual or classical polytrauma patient has multiple injuries, some of which may require withholding the prophylactic dose for 24-48 h while others may require therapeutic doses for saving the patient's limbs and life. In this paper, we would like to describe the case of a patient who represents a classic ex- 
Fig. 1. a CT abdominal angiogram crosssectional view. b CT abdominal angiogram coronal view.
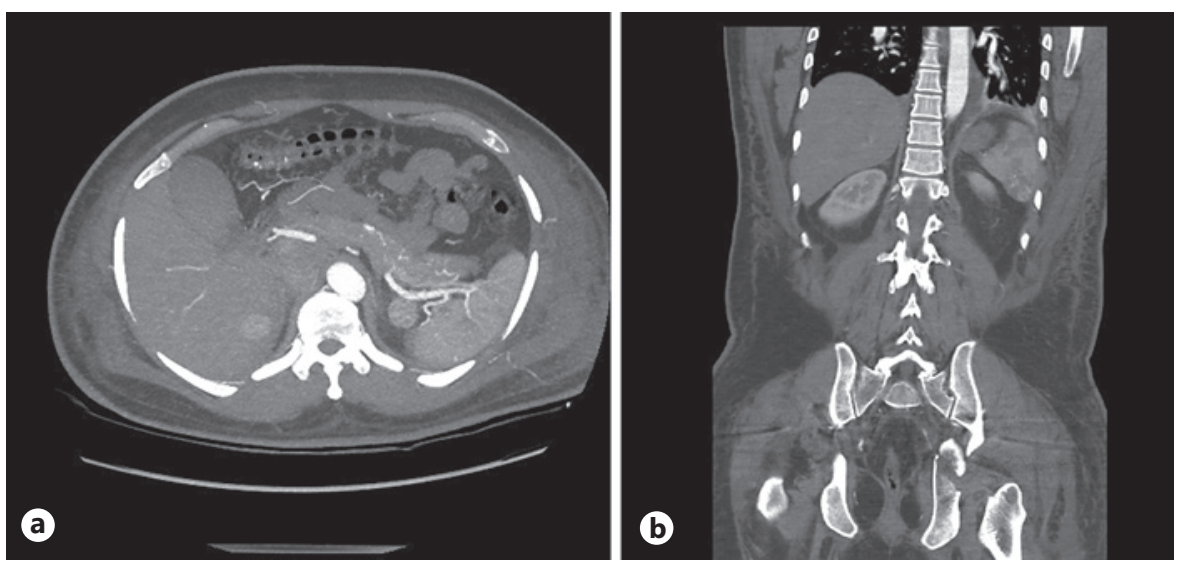

ample of the dilemma encountered in the polytrauma cohort; a patient who needs TAC to save his limb but has other concomitant injuries that warrant a more calculated approach.

\section{Case Report/Case Presentation}

A 45-year-old male was involved in a motor vehicle collision while riding a motor bicycle. He was initially taken to a peripheral hospital where he had a polytrauma computed tomography (CT) scan that showed a comminuted pelvic fracture (iliac bone, ala sacrum, acetabulum pubic bone, superior and inferior pubic rami), a grade III splenic injury with no significant intraperitoneal freemoving fluid collection, a retroperitoneal hematoma, and a left comminuted open type $3 \mathrm{C}$ left tibia and fibula shaft fracture. CT angiogram of the lower limbs was done and showed a left popliteal artery segment 2 injury. There was refilling to the posterior tibial and peroneal arteries, with acceptable signals above the ankle level.

He underwent open reduction internal fixation of the left tibial bicondylar displaced open fracture. Three days later, he was transferred to our level 1 trauma center for further management and treatment by orthopedic and vascular surgery teams for his pelvic fracture and popliteal injuries.

On arrival in the early hours of the morning to the Emergency Room (ER), the patient was assessed by our emergency staff and was found to have an ex-fix applied to the left lower limb for the tibia and fibula shaft fractures. Right lower limb superficial pulses were found on digital and Doppler examinations. Left lower limb superficial pulses were found on Doppler examination. The patient was booked for surgery in the day for left popliteal artery exploration. His hemoglobin $(\mathrm{Hb})$ on arrival was $93 \mathrm{~g} / \mathrm{L}$. However, in the morning, the patient developed edema of the left lower extremity. His foot was cold, and he had delayed capillary refill and absent pulses and signals in the pedal arteries. Thus, he was taken to theater for a limb-saving procedure. By then, his $\mathrm{Hb}$ had fallen to $88 \mathrm{~g} / \mathrm{L}$.

\section{Intraoperative Findings and Procedure}

A left popliteal artery level 2 exposure was done and was found to be completely cut. So, a vein interposition graft with end-to-end anastomosis proximally and distally was performed. There was also a complete cut of left tibialis posterior for which an end-to-end anastomosis was done. Finally, a left medial fasciotomy was also done. Orthopedics later joined and removed the pervious ex-fix on the left leg, debrided the wound, and placed a new ex-fix in its place. The pelvic fracture was stabilized in an ex-fix.

\section{Postoperative}

One day after left popliteal artery repair (4 days following the accident), the vascular team wanted to start the patient on TAC (low-molecular-weight heparin 6,000 IU twice daily) to preserve the graft and save the limb. His prothrombin time (PT) was 15.0 $\mathrm{s}$ (reference range 11-14 s), activated partial thromboplastin time (APTT) was $37.8 \mathrm{~s}$ (reference range 28.0-41.0 s), and international normalized ratio (INR) was 1.17 (reference range 0.8 1.1).

In view of his grade III splenic injury, our general surgery team was consulted. We ordered a CT abdominal angiogram, shown in Figure 1a and $b$, which showed no evidence of contrast extravasation from the splenic injury, no pseudoaneurysm, and no increase in the hemoperitoneum. Thus, clearance was given to start the patient on TAC accompanied by serial abdominal examinations and close monitoring of his $\mathrm{Hb}$. Repeat PT was $15.3 \mathrm{~s}$, APTT $37.6 \mathrm{~s}$, and INR 1.20 . We continued to closely observe the patient as we managed his grade III splenic injury conservatively. He required another operation (realignment of external fixator and wound debridement) by orthopedic and vascular teams which was done successfully.

As the patient was stable vitally and clinically with stable $\mathrm{Hb}$, he was discharged from general surgery team (10 days after starting LMWH), while he continued treatment of his fractures and vessel injuries by orthopedic and vascular teams. He underwent several operations later on during his hospital stay including multiple wound debridement, fixation for acetabulum fracture, and re-adjustment of fixator. Finally, he required a left below knee amputation which was carried out not because of graft failure but rather due to the big composite defect in his left leg for which a fibula flap was not feasible, and thus the alternative for best fast functional outcome was a below knee amputation and a prosthetic fitting thereafter. He was discharged from the hospital in good condition around 2 months after admission, with scheduled follow-ups by the orthopedic team. 


\section{Discussion/Conclusion}

This case has tackled a challenge that is yet to be resolved: the safety of starting therapeutic (high-dose) anticoagulation therapy in patients with high-grade abdominal solid organ injuries. Our case is the first to describe the use of therapeutic, rather than prophylactic, anticoagulation in a patient with multiple injuries including a grade III splenic injury, who required therapeutic LMWH for preserving his limb.

On literature review, we found a research study that looked at the safety of early ( $\leq 48 \mathrm{~h})$, intermediate (48-72 $\mathrm{h})$, and late $(\geq 72 \mathrm{~h})$ VTE prophylaxis in patients with blunt abdominal solid organ injury managed nonoperatively. The study arrived to a conclusion that early enoxaparin-based anticoagulation (LMWH 3,000 IU subcutaneously every $12 \mathrm{~h}$ ) may be a safe option in trauma patients with blunt solid organ injury, and that there is no significant correlation between early anticoagulation and the development of bleeding complications in such patients [5].

Regarding patients with traumatic brain injury (TBI), a multicenter prospective study focused on anticoagulation therapy initiation in patients with TBI and concluded that patients who received anticoagulation therapy later on after the injury had lower risk of clinically significant TBI progression and less chances of requiring neurosurgical intervention than those who had received anticoagulation therapy soon after the injury (11 days vs. 4.5 days, $p=0.015$ ) [8].

On the other hand, Shahan et al. [9] looked retrospectively at patients admitted with blunt cerebrovascular injury (BCVI) and concomitant TBI and/or solid organ injury (SOI) over 6 years and reported that early initiation of antithrombotic therapy (antiplatelet or heparin therapy, as early as possible upon diagnosis of BCVI) for patients with BCVI and concomitant TBI and/or SOI does not lead to worsening of TBI or SOI above baseline and, in fact, can prevent stroke-related morbidity and mortality in these patients [9].

Another research was carried out to evaluate the safety of initiating TAC for patients with TBI (within 60-day after the injury period). Seventy-two patients out of 3,355 patients with TBI were given TAC. None of the study patients developed signs of neurologic deterioration due to TAC. Six patients had progression of hemorrhagic TBI on repeat head CT, those aged 65 years and above being the most commonly affected by hemorrhagic progression of TBI (odds ratio:15.2; 95\% confidence interval, 1.1-212.7; $p=0.04)$. However, the data in this study are preliminary, and further prospective studies are needed on this topic [10].

The nonoperative management (NOM) of blunt liver/ spleen $(\mathrm{L} / \mathrm{S})$ injuries in patients with concomitant blunt aortic injuries (BAI) was studied by Santaniello et al. [6], with the conclusion that NOM of patients with grade I or II L/S injury who undergo systemic anticoagulation with heparin for repair of BAI is safe and results in a similar transfusion rate to patients with $\mathrm{BAI}$ without $\mathrm{L} / \mathrm{S}$ injuries. Such patients do not require laparotomy before BAI repair using partial bypass. This study therefore proved that low-grade liver and spleen injuries can be anticoagulated acutely, with minimal risk of delayed hemorrhage [6].

Our patient, on the other hand, had a grade III splenic injury and yet was therapeutically anticoagulated with high-dose LMWH without bleeding complications. Updated guidelines on the pharmacologic prophylaxis for VTE prevention in trauma patients have been published by the Western Trauma Association (WTA) Algorithms committee in 2020 [7]. These guidelines focused on administering prophylactic doses of anticoagulation to trauma victim patients.

The algorithm mentioned that patients with solid organ injury who received early pharmacologic prophylaxis had lower DVT and pulmonary embolism rates without increased risk of failure of NOM, bleeding complications, or mortality, with similar risks when prophylaxis was started within 24 or $48 \mathrm{~h}$.

Regarding patients with TBI, pharmacologic prophylaxis may be delayed depending on the type of TBI. Patients with cerebral contusion, localized petechial hemorrhages, or diffuse axonal injury can be given pharmacologic prophylaxis without delay. If prophylaxis is delayed due to the patient having other types of TBI, follow-up CT is an important indicator for when to initiate pharmacologic prophylaxis, with the need to further delay prophylaxis for patients with CT evidence of TBI progression.

It was noted that trauma centers which provide prophylaxis within $24 \mathrm{~h}$ after TBI have significantly lower rates of VTE with no difference in rates of late neurosurgical intervention [11-13]. However, no algorithms or case reports have been published describing TAC for trauma patients with solid organ injuries.

A point for discussion is the traumatic coagulopathy in polytrauma patients. Trauma-induced coagulopathy (TIC) is a clinical syndrome featuring coagulopathy occurring in the early stage of trauma, caused by activation of coagulation, fibrinolytic, and anticoagulation pathways due to several factors including tissue injury and bleeding [14]. Other terms used to refer to this condition 
include acute traumatic coagulopathy, early coagulopathy of trauma, and the acute coagulopathy of traumashock [15-17].

It may be the result of physiologic derangements such as acidosis, hypothermia, or hemodilution due to fluid or blood administration. However, the current opinion is that it is an endogenous acute coagulopathy existing independently and is different from coagulation disorders caused by acquired factors [14].

In the early hours of TIC development, hypocoagulability is typically present with prolonged PT and PTT, resulting in bleeding. Later on, a hypercoagulable state predominates associated with venous thromboembolism and multiple organ failure [18]. The presence of TIC has been demonstrated to be an independent predictor for increased organ dysfunction, infection, and overall mortality $[19,20]$.

The so-called lethal triad which features acidosis, hypothermia, and coagulopathy is thought to aggravate TIC [14]. Our patient's coagulation profile was closely monitored, and he was safely treated without going into this triad. The concept of post-traumatic TIC is still not well understood, and there is a lack of international standards for its diagnosis.

\section{Conclusion}

Our patient was started on TAC with LMWH 4 days after the injury, as requested by the vascular team. His grade III splenic injury was managed conservatively, with no signs of extravasation or pseudoaneurysm on followup CT abdominal angiogram.

In summary, we followed a simple approach to the polytrauma patient needing TAC with high-dose LMWH after opting for NOM:

1. Rule out any on-going bleeding.

2. Rule out the presence of traumatic pseudoaneurysms.

3 . In the event of finding a pseudoaneurysm, embolization should be carried out by Interventional Radiology.

\section{References}

Therapeutic Anticoagulation with HighGrade Abdominal Solid Organ Injury
4. Admit the patient in a monitored setting.

5. Serial Hb levels (6-8 hourly).

This case report is a call for extensive research to be done on this matter, to study the role of TAC in patients with high-grade abdominal solid organ injuries managed nonoperatively, in order to establish protocols for the optimal time and dose of anticoagulation that can be safely used in such patients and to report the possible expected complications and how to manage them appropriately.

\section{Statement of Ethics}

This case report study complies with the guidelines for human studies and was done ethically in accordance with the World Medical Association Declaration of Helsinki. Ethical approval is not required in accordance with the Dubai Health Authority Ethics Committee policies. Written informed consent was obtained from the patient for publication of this case report and any accompanying images.

\section{Conflict of Interest Statement}

The authors have no conflicts of interest to declare.

\section{Funding Sources}

The authors did not receive any funding.

\section{Author Contributions}

Y.H.A. and A.H.S. contributed to writing the manuscript and data collection. Y.H.E.T. and A.K.Y. contributed to study design and review of the final report.

\section{Data Availability Statement}

All data generated during this case report study are included in this article. Further enquiries can be directed to the corresponding author.
1 Key NS, Khorana AA, Kuderer NM, Bohlke K, Lee AY, Arcelus JI, et al. Venous thromboembolism prophylaxis and treatment in patients with cancer: ASCO clinical practice guideline update. J Clin Oncol. 2020;38(5): 496-520.

2 Ressel GW; American College of Obstetricians and Gynecologists. ACOG practice bulletin on preventing deep venous thrombosis and pulmonary embolism. Am Fam Physician. 2001 Jun 1;63(11):2279-80.
3 Flevas DA, Megaloikonomos PD, Dimopoulos L, Mitsiokapa E, Koulouvaris P, Mavrogenis AF. Thromboembolism prophylaxis in orthopaedics: an update. EFORT Open Rev. 2018;3(4):136-48.

4 Bartlett MA, Mauck KF, Daniels PR. Prevention of venous thromboembolism in patients undergoing bariatric surgery. Vasc Health Risk Manag. 2015;11:461-77. 
5 Joseph B, Pandit V, Harrison C, Lubin D, Kulvatunyou N, Zangbar B, et al. Early thromboembolic prophylaxis in patients with blunt solid abdominal organ injuries undergoing nonoperative management: is it safe? Am J Surg. 2015 Jan;209(1):194-8.

6 Santaniello JM, Miller PR, Croce MA, Bruce L, Bee TK, Malhotra AK, et al. Blunt aortic injury with concomitant intra-abdominal solid organ injury: treatment priorities revised. J Trauma. 2002;53:442-5.

7 Ley EJ, Brown CVR, Moore EE, Sava JA, Peck $\mathrm{K}$, Ciesla DJ, et al. Updated guidelines to reduce venous thrombembolism in trauma patients: a Western Trauma Association critical decisions algorithm. J Trauma Acute Care Surg. 2020;89(5):971-81.

8 Matushima K, Leichtle SW, Wild J, Young K, Chang G, Demetriades D, et al. Anticoagulation therapy in patients with traumatic brain injury: an Eastern Association for the surgery of trauma multicenter prospective study. Surgery. 2021;169:470-6.

9 Shahan CP, Magnotti LJ, McBeth PB, Weinberg JA, Croce MA, Fabian TC. Early antithrombotic therapy is safe and effective in patients with blunt cerebrovascular injury and solid organ injury or traumatic brain injury. J Trauma Acute Care Surg. 2016;81(1):173-7.
10 Matsushima K, Inaba K, Cho J, Mohammed $\mathrm{H}$, Herr K, Leichtle S, et al. Therapeutic anticoagulation in patients with traumatic brain injury. J Surg Res. 2016;205:186-91.

11 Byrne JP, Mason SA, Gomez D, Hoeft C, Subacius $\mathrm{H}$, Xiong $\mathrm{W}$, et al. Timing of pharmacologic venous thromboembolism prophylaxis in severe traumatic brain injury: a propensitymatched cohort study. J Am Coll Surg. 2016; 223(4):621-31.e5.

12 Störmann P, Osinloye W, Freiman TM, Seifert V, Marzi I, Lustenberger T. Early chemical thromboprophylaxis does not increase the risk of intracranial hematoma progression in patients with isolated severe traumatic brain injury. World J Surg. 2019;43(11):2804-11.

13 Meyer RM, Larkin MB, Szuflita NS, Neal CJ, Tomlin JM, Armonda RA, et al. Early venous thromboembolism chemoprophylaxis in combat-related penetrating brain injury. J Neurosurg. 2017;126(4):1047-55.

14 Peng N, Su L. Progresses in understanding trauma-induced coagulopathy and the underlying mechanism. Chin J Traumatol. 2017; 20(3):133-6.
15 Brohi K, Singh J, Heron M, Coats T. Acute traumatic coagulopathy. J Trauma. 2003 Jun; 54(6):1127-30.

16 Hess JR, Brohi K, Dutton RP, Hauser CJ, Holcomb JB, Kluger Y, et al. The coagulopathy of trauma: a review of mechanisms. J Trauma. 2008;65:748.

17 Jimenez JCB, de La Pena JL, Teheran RM, Orozco A. Early coagulopathy in trauma: do coagulopathic patients reach the operating room? Rev Colomb Anestesiol. 2010;38(4): 510-25.

18 Moore EE, Moore HB, Kornblith LZ, Neal MD, Hoffman M, Mutch NJ, et al. Traumainduced coagulopathy. Nat Rev Dis Primers. 2010;7:30.

19 Cohen MJ, Call M, Nelson M, Calfee CS, Esmon CT, Brohi K, et al. Critical role of activated protein $C$ in early coagulopathy and later organ failure, infection and death in trauma patients. Ann Surg. 2012 Feb;255(2): 379-85.

20 MacLeod JB, Lynn M, McKenney MG, Cohn SM, Murtha M. Early coagulopathy predicts mortality in trauma. J Trauma. 2003 Jul;55(1): $39-44$. 